nephron

Practice
Nephron 2018;140:134-139

DOI: $10.1159 / 000490006$
Received: April 30, 2018

Accepted after revision: May 12, 2018

Published online: June 5, 2018

\title{
Quantitative Large-Scale Three-Dimensional Imaging of Human Kidney Biopsies: A Bridge to Precision Medicine in Kidney Disease
}

\author{
Seth Winfree ${ }^{a, b}$ Pierre C. Dagher ${ }^{a, b, e}$ Kenneth W. Dunn ${ }^{a}$ Michael T. Eadon $^{a}$ \\ Michael Ferkowicz ${ }^{c}$ Daria Barwinska ${ }^{a}$ Katherine J. Kelly ${ }^{a}$ Timothy A. Sutton $^{a}$ \\ Tarek M. El-Achkara, b, d, e \\ aDepartment of Medicine, Division of Nephrology, Indiana University School of Medicine, Indianapolis, IN, USA; \\ ${ }^{b}$ Department of Integrative and Cellular Physiology, Indiana University, Indianapolis, IN, USA; ${ }^{\circ}$ Department of \\ Surgery, Indiana University, Indianapolis, IN, USA; ${ }^{\mathrm{d}}$ Department of Anatomy and Cell Biology, Indiana University \\ School of Medicine, Indianapolis, IN, USA; ' Indianapolis VA Medical Center, Indianapolis, IN, USA
}

\section{Keywords \\ Three-dimensional imaging · Kidney biopsy · \\ Tissue cytometry}

\begin{abstract}
Kidney biopsy remains the gold standard for uncovering the pathogenesis of acute and chronic kidney diseases. However, the ability to perform high resolution, quantitative, molecular and cellular interrogation of this precious tissue is still at a developing stage compared to other fields such as oncology. Here, we discuss recent advances in performing large-scale, three-dimensional (3D), multi-fluorescence imaging of kidney biopsies and quantitative analysis referred to as 3D tissue cytometry. This approach allows the accurate measurement of specific cell types and their spatial distribution in a thick section spanning the entire length of the biopsy. By uncovering specific disease signatures, including rare occurrences, and linking them to the biology in situ, this approach will enhance our understanding of disease pathogenesis. Furthermore, by providing accurate quantitation of
\end{abstract}

cellular events, 3D cytometry may improve the accuracy of prognosticating the clinical course and response to therapy. Therefore, large-scale 3D imaging and cytometry of kidney biopsy is poised to become a bridge towards personalized medicine for patients with kidney disease.

(c) 2018 S. Karger AG, Basel

\section{Introduction}

Kidney disease can be broadly divided into acute and chronic kidney disease $[1,2]$. The diagnosis of kidney damage relies primarily on the history and laboratory testing for filtration markers such as serum cre-

Contribution from the AKI and CRRT 2018 Symposium at the 23rd International Conference on Advances in Critical Care Nephrology, Manchester Grand Hyatt, San Diego, CA, USA, February 26-March 1, 2018. This symposium was supported in part by the NIDDK funded University of Alabama at Birmingham-University of California San Diego O'Brien Center for Acute Kidney Injury Research (P30DK079337).

\section{KARGER}

(c) 2018 S. Karger AG, Basel

E-Mail karger@karger.com

www.karger.com/nef
Dr. Seth Winfree and Dr. Tarek M. El-Achkar

Indiana University School of Medicine

950 West Walnut Street

Indianapolis, IN 46202 (USA)

E-Mail winfrees@iu.edu, telachka@iu.edu 
atinine. However, a kidney biopsy is frequently performed when the etiology of kidney dysfunction is uncertain, when disease-modifying agents with serious side effects are to be considered, and less frequently for prognostication purposes [3, 4]. Nevertheless, a kidney biopsy is not without risks, such as bleeding and less commonly loss of the kidney, and the risks are significantly higher in sicker patients [5-7]. Consequently, such biopsies are considered by the patients (who undergo the procedure), nephrologists (who perform the procedure) and pathologists (who process and interpret the tissue) as precious and irreplaceable specimens that should provide a maximal amount of essential information.

The ideal kidney biopsy procedure will yield $2-3$ cores that span the kidney cortex and contain a threshold number of glomeruli needed to minimize sampling bias. The cores are processed for light microscopy, direct immunofluorescence staining for immune complex deposits, and electron microscopy [8]. The pathology interpretation relies heavily on the experience of the pathologists, and the report frequently includes semi-quantitative assessment of the various stains, which are reported from fields within thin sections imaged in 2 dimensions. This standard approach has been the cornerstone of diagnosis and is still widely used. However, technological advances in cellular and molecular interrogations of tissues provide an impetus to consider the breadth of information that could be extracted by applying these techniques to a kidney biopsy. These timely advances coincide with a national interest in not only looking at disease subcategories [1] but also uncovering patient-specific factors that could be key in determining disease pathogenesis, prognosis and response to therapy [9].

Therefore, several factors will usher the interpretation of kidney biopsies into the era of precision medicine. These efforts have already started, driven by pathologists and renal investigators through adoption in large consortial trials such as NEPTUNE, whereby whole slide imaging and assisted quantitative interpretation using digital pathology software have been implemented $[10,11]$. Despite the two-dimensional aspect of this approach, which still has limitations, this is considered a significant advancement that will have a profound impact. In this brief commentary, we will discuss advances in large-scale, multi-fluorescence, three-dimensional (3D) imaging of kidneys biopsies for quantitative and exploratory analysis using tissue cytometry software. This approach is another step forward that will advance the field of cellular and molecular interrogation of kid- ney biopsies and empower pathologists and nephrologists to better understand, diagnose and prognosticate kidney disease.

\section{Why Large-Scale, 3D Multi-Fluorescence Confocal Imaging?}

First, let us define what is meant by large-scale multifluorescence imaging. This signifies that confocal imaging spans the entire length of the biopsy, which is typically $1-2 \mathrm{~cm}$ in length $[12,13]$. The $3 \mathrm{D}$ aspect of this approach requires imaging a volume thick enough to capture whole structures, balanced by the need to allow homogenous penetration of stains, antibodies and imaging light across the entire thickness. A thickness of 50-100 $\mu \mathrm{m}$ typically satisfies these requirements [12]. To capture the image volume at a resolution high enough to distinguish and segment individual cells, imaging is typically performed at least with a $20 \times$ objective. Overlapping image volumes are then digitally stitched to produce a mesoscale 3D image volume [12]. The advantages of this approach can be summarized as follows [13]:

(a) Achieving accurate spatial characterization and relationship between structures. This can be easily demonstrated in examples where entire structures can be missed when looking at a single optical slice compared to an image volume spanning an entire thickness. The $\mathrm{z}$ dimension exposes the complex relationship between cells and structures beyond a view from a single plane.

(b) Improving data precision by capturing uneven distribution of cell surface markers across several optical planes.

(c) Performing accurate quantitation of cell distribution and density in situ using 3D tissue cytometry (discussed below).

(d) Because of the scale of imaging and its quantitative focus, this methodology will detect rare events and patient-specific changes, which are key features of a precision medicine approach.

\section{Optical Sectioning Modalities Enabling Large-Scale 3D Imaging}

There are 3 main imaging modalities that make it possible to implement this approach: confocal microscopy, multi-photon microscopy and selective plan illumination (or light sheet) microscopy [14-16]. Each modality has some advantage over the others, and we refer the reader 
to a more in-depth review by our group where this topic is discussed in more detail [12]. We commonly use confocal microscopy because of the ease and feasibility of performing spectral scanning and unmixing, thereby enabling the detection of up to 8 labels simultaneously. Indeed, using multiple labels for tubular and immune cells may uncover different sub-populations of cells or detect new associations between various cell types.

\section{D Tissue Cytometry: The Equivalent of Flow Cytometry in Intact Tissues with Perks!}

The pinnacle of this approach must be defined by the ability to perform accurate quantitation of specific cell types in intact tissue, their spatial localization and distribution, and relationship to other structures. Flow cytometry analysis, despite its limitations when applied to tissues (as far as loss of spatial parameters and bias of dissociation), presents a user-friendly way to quantitate cells through scatter plot visualization scheme of cells based on staining intensity. Gating of specific clusters of cells allows the quantitation of subpopulations of interest. This philosophy of analysis guided our approach in designing a tool that enables analysis like flow cytometry, but in intact tissue with preserved spatial parameters that are captured by multi-fluorescence 3D imaging. Other important considerations were to incorporate the analysis a single platform, easily adaptable to current practices, allowing a seamless, bi-directional workflow of image processing, segmentation, analysis and direct interaction with the image volume.

\section{Volumetric Tissue Exploration and Analysis: A Unique Tool for 3D Tissue Cytometry}

We devised volumetric tissue exploration and analysis (VTEA) as a solution for 3D tissue cytometry (Fig. 1). VTEA is a plug in to ImageJ, a commonly used tool for image visualization and analysis. In a single workspace, VTEA allows image visualization, processing, segmentation and analysis, through a bidirectional workflow that also facilitates back-and-forth interactivity between the image and the analysis. Each cell within a volume is identified using a $3 \mathrm{D}$ segmentation strategy that uses the nucleus as a fiduciary for the cell (Fig. 1A). Registering spatial coordinates and various measurements of fluorescence label intensities within a defined area around each nucleus will determine various attributes associated with each cell and define a set of variables that make up a feature space. Plotting these cells in a scatter with 2 or 3 dimensions will allow the identification of cells of interest based on attributes such as fluorescence intensity. Gating of cells with a high expression of a specific marker will identify these cells on the image, along with the associated statistics, such as absolute number of cells counted and percentage of the total cells (Fig. 1B). Density determination is an inherently derived statistic, since the volume of the images or any region of interest is also available. The gated cells are directly identified in the image with nuclear overlays throughout the thickness of the volume, which serves as a direct method of validating the gates, and links the cell population of interest to the biology in situ. Identification of regions of interest within the image will also highlight, in the scatter plot, all the cells present in that region, with the corresponding statistics. This approach will allow interaction between the image and scatter plot, thereby facilitating interactive exploration of the image volume.

\section{Application of Large-Scale, 3D Cytometry with VTEA to Kidney Biopsies}

The applicability of 3D cytometry can be better appreciated in a clinical context. Diabetic nephropathy is a leading cause of chronic and end-stage kidney disease [17]. The diagnosis of diabetic nephropathy is made on clinical grounds, and patients are rarely biopsied unless the diagnosis is uncertain. Even if a biopsy is obtained, the ability to predict a progressive decline in kidney function based on the pathological assessment of the biopsy is limited [18]. Therefore, there is a dire need to improve the ability to perform cellular and molecular interrogations that not only will help elucidate the pathogenesis of disease but also help prognosticate and guide the response to therapy.

We recently demonstrated the feasibility of largescale, 3D tissue cytometry in patients with diabetic nephropathy and showed the usefulness of such an approach when linked to longitudinal clinical data [13]. We showed how this approach can detect an increase in neutrophil infiltration in a patient who rapidly progressed to ESRD compared to a patient with a slower rate of progression. The patchy nature of such neutrophil infiltration may have been easily missed if looking in a thin section or in 3D [13]. Therefore, our robust approach allows the detection of less common events, which may be crucial in uncovering the pathogenesis of disease, or in delineating patient-specific factors that modify the course 


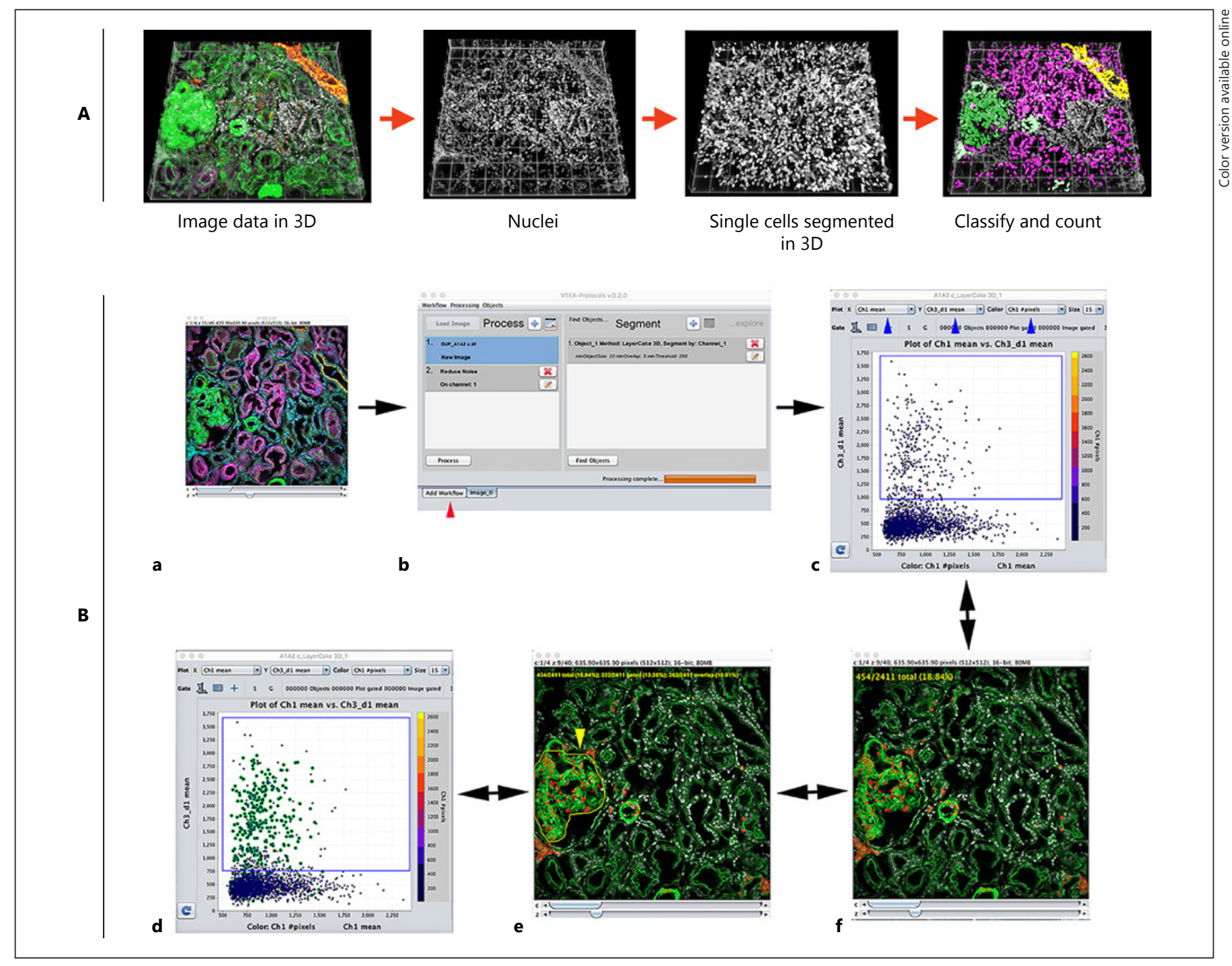

Fig. 1. Volumetric tissue exploration and analysis (VTEA) tool for 3D tissue cytometry. A Design of a workflow to perform cytometry on 3D image volumes, by using the nuclei as fiduciaries for all cells present in the volumes. B Demonstration of tissue cytometry workflow using VTEA. a The image volume loaded into ImageJ/ FIJI. b Basic image preprocessing and 3D segmentation are managed through the VTEA plugin GUI. c Segmented 3D nuclei are quantified by clicking the Find Objects button, which generates a scatterplot GUI, in which each dot represents measured parameters for a single cell. Up to 3 measurement parameters can be selected for simultaneous display ( $\mathrm{x}$-axis, $\mathrm{y}$-axis, and color) via a

of disease or response to therapy. Those initial observations are currently being tested now in a larger cohort of patients with diabetes to link immune cell distribution and abundance to the clinical course. This example also underscores the importance of developing biobanks of kidney biopsies linked to a longitudinal clinical database. menu interface (blue arrows). d Rectangles can be drawn on the scatterplot to identify subpopulations of cells (gating), which are highlighted as nuclear overlays in the image volume to identify their spatial distribution (shown in red). e Free-hand regions-ofinterests can also be drawn directly on the image volume to isolate particular structures or areas (in this case, a yellow region is drawn around a glomerulus), which generates a new scatterplot (seen in f) displaying quantifications of cells from the demarcated region (shown here as green-outlined dots). Part (B) is used with permission from the journal of the American Society of Nephrology (JASN July 2017 vol. 28 no. 7 p2108-p2118).

By linking finite cellular and molecular variables to the clinical course using large-scale, 3D tissue cytometry, this model showcases a significant step forward in implementing a personalized medicine approach to patients with kidney disease. 


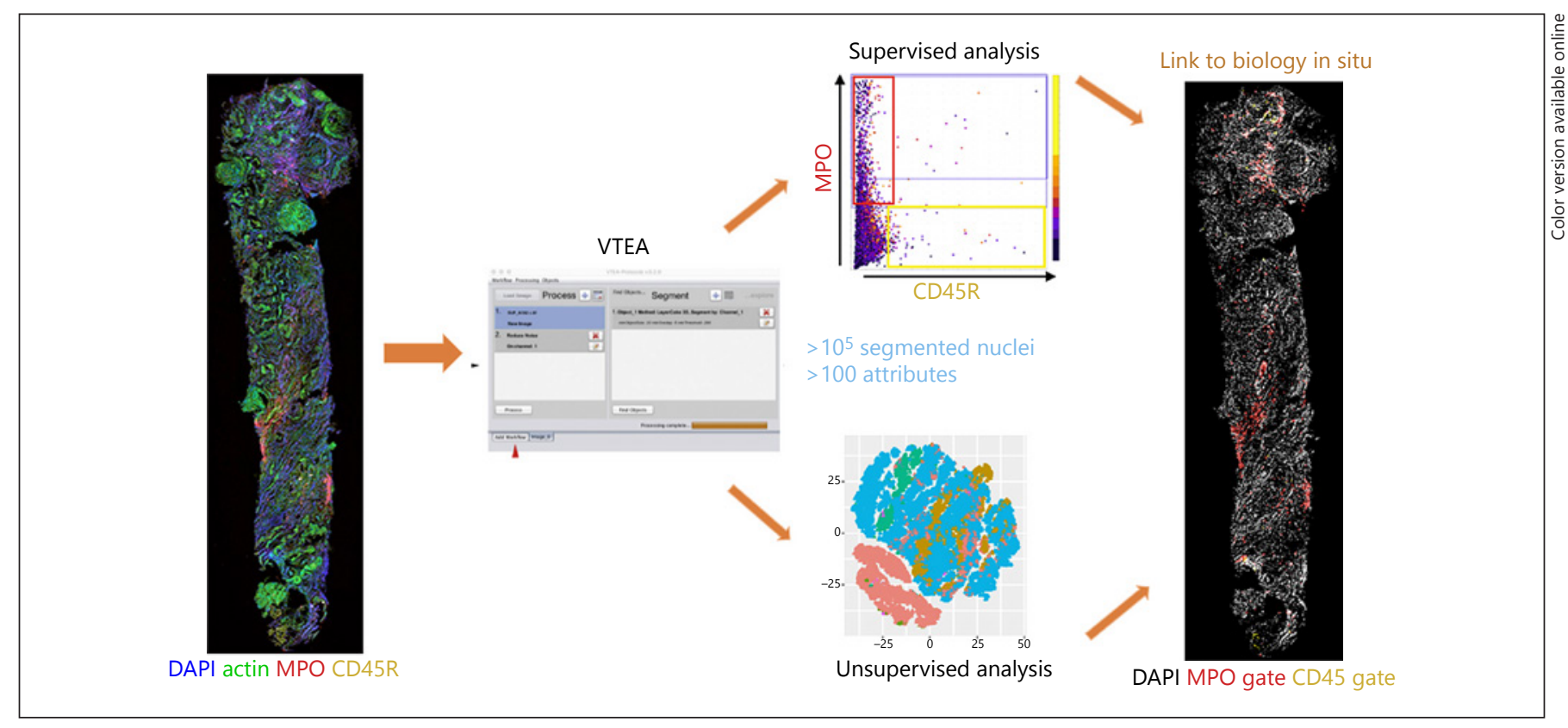

Fig. 2. Application of large-scale 3D imaging and tissue cytometry analysis to human kidney biopsies. This workflow integrates our current approach and the future vision of how to apply this technology to interrogate kidney biopsies. We envision that big data will be generated, and that not only supervised analysis can be performed but also unsupervised analyses using machine learning tools. The power of tissue cytometry stems from the fact that all analyses are linked to the image volume, that is, to the biology in situ. VTEA, Volumetric tissue exploration and analysis.

\section{Conclusions and Future Directions}

We present in this mini-review an overview on largescale 3D imaging and tissue cytometry and its application to interrogate human kidney biopsies. We foresee that the amount and type of data extracted will become increasingly complex. Already, the current feature space is generating over 70 attributes per cell. Applying an analysis to thousands of cells that account for this complexity (beyond examining 2 or 3 variables at a time, such as with scatter plots) is daunting. In addition, some association between variables may not be intuitive and will not be readily uncovered by a supervised type of analysis. Therefore, analyzing multi-parametric data generated by this approach will need to incorporate both unsupervised methodologies (machine learning), in addition to the supervised strategies that we described. Our vision and direction for large-scale 3D imaging and tissue cytometry is summarized in Figure 2.

In conclusion, by uncovering specific and novel disease signatures, including rare occurrences, and linking them to the biology in situ, large-scale, 3D imaging and tissue cytometry will enhance our understanding of disease pathogenesis and likely improve the accuracy of prognosticating the clinical course and response to ther apy. This approach will become an important asset to establish a personalized medicine approach for patients with kidney disease.

\section{Acknowledgements}

Funding support was received from NIH-NIDDK- DK076169 Diacomp (T.M.E-A. and P.C.D.), Indiana O'Brien Center for Advanced Microscopic Analysis (NIH-NIDDK P30 DK079312).

\section{Disclosure Statement}

The authors have no conflicts of interest to declare.

References

Winfree et al.
1 Hall YN, Himmelfarb J: The CKD classification system in the precision medicine era. Clin J Am Soc Nephrol 2017;12:346-348.

2 Zuk A, Bonventre JV: Acute kidney injury. Annu Rev Med 2016;67:293-307.

-3 Iseki K, Miyasato F, Uehara H, Tokuyama K, Toma S, Nishime K, Yoshi S, Shiohira Y, Oura T, Tozawa M, Fukiyama K: Outcome study of renal biopsy patients in Okinawa, Japan. Kidney Int 2004;66:914-919. 
4 Madaio MP: Renal biopsy. Kidney Int 1990; 38:529-543.

5 Atwell TD, Spanbauer JC, McMenomy BP, Stockland AH, Hesley GK, Schleck CD, Harmsen WS, Welch TJ: The timing and presentation of major hemorrhage after 18,947 image-guided percutaneous biopsies. AJR Am J Roentgenol 2015;205:190-195.

-6 Corapi KM, Chen JL, Balk EM, Gordon CE: Bleeding complications of native kidney biopsy: a systematic review and meta-analysis. Am J Kidney Dis 2012;60:62-73.

7 Whittier WL, Korbet SM: Timing of complications in percutaneous renal biopsy. J Am Soc Nephrol 2004; 15:142-147.

8 Walker PD: The renal biopsy. Arch Pathol Lab Med 2009;133:181-188.

$\checkmark 9$ Gluck C, Ko YA, Susztak K: Precision medicine approaches to diabetic kidney disease: tissue as an issue. Curr Diab Rep 2017;17: 30.

10 Barisoni L, Troost JP, Nast C, Bagnasco S, Avila-Casado C, Hodgin J, Palmer M, Rosenberg A, Gasim A, Liensziewski C, Merlino L, Chien HP, Chang A, Meehan SM, Gaut J, Song P, Holzman L, Gibson D, Kretzler M,
Gillespie BW, Hewitt SM: Reproducibility of the NEPTUNE descriptor-based scoring system on whole-slide images and histologic and ultrastructural digital images. Mod Pathol 2016;29:671-684.

11 Barisoni L, Nast CC, Jennette JC, Hodgin JB, Herzenberg AM, Lemley KV, Conway CM, Kopp JB, Kretzler M, Lienczewski C, Avila-Casado C, Bagnasco S, Sethi S, Tomaszewski J, Gasim AH, Hewitt SM: Digital pathology evaluation in the multicenter Nephrotic Syndrome Study Network (NEPTUNE). Clin J Am Soc Nephrol 2013; 8:1449-1459.

12 Winfree S, Ferkowicz MJ, Dagher PC, Kelly KJ, Eadon MT, Sutton TA, Markel TA, Yoder MC, Dunn KW, El-Achkar TM: Large-scale 3-dimensional quantitative imaging of tissues: state-of-the-art and translational implications. Transl Res 2017;189:1-12.

13 Winfree S, Khan S, Micanovic R, Eadon MT, Kelly KJ, Sutton TA, Phillips CL, Dunn KW, El-Achkar TM: Quantitative three-dimensional tissue cytometry to study kidney tissue and resident immune cells. J Am Soc Nephrol 2017;28:2108-2118.
4 Ustione A, Piston DW: A simple introduction to multiphoton microscopy. J Microsc 2011; 243:221-226

15 Santi PA: Light sheet fluorescence microscopy: a review. J Histochem Cytochem 2011;59: 129-138.

16 Chung K, Wallace J, Kim SY, Kalyanasundaram S, Andalman AS, Davidson TJ, Mirzabekov JJ, Zalocusky KA, Mattis J, Denisin AK, Pak S, Bernstein H, Ramakrishnan C, Grosenick L, Gradinaru V, Deisseroth K: Structural and molecular interrogation of intact biological systems. Nature 2013;497:332337.

17 Tuttle KR, Bakris GL, Bilous RW, Chiang JL, de Boer IH, Goldstein-Fuchs J, Hirsch IB, Kalantar-Zadeh K, Narva AS, Navaneethan SD, Neumiller JJ, Patel UD, Ratner RE, WhaleyConnell AT, Molitch ME: Diabetic kidney disease: a report from an ADA Consensus Conference. Am J Kidney Dis 2014;64:510-533.

18 An Y, Xu F, Le W, Ge Y, Zhou M, Chen H, Zeng C, Zhang H, Liu Z: Renal histologic changes and the outcome in patients with diabetic nephropathy. Nephrol Dial Transplant 2015;30:257-266. 